\title{
Orthodontic Treatment a Boon or Bane to TMJ
}

\author{
Dr. ThomasJohn, Dr. AbhishekBansal, Dr. AsmaFatima, Dr. Afshan, \\ Dr. VakiChinde, Dr. AsiyaFatima
}

\begin{abstract}
TMJ disorders are a complication in itself. The orthodontic treatment is considered sometimes as a remedy for the TMJ disorders whereas other few times the same is considered as culprit. The common problems encountered in the clinical practice are Class II cases. Therefore the treatment plans for the cases are taken into consideration for the study. The main aim of the study is to determine, does Orthodontic treatment will cause TMD disorder or an remedy for TMD disorders.A Sample of 100 Patients between the age group of 10-22 yrs were taken for the Study, divided into two groups depending on the case and treatment plan. It was observed that during the course of the treatment patients of $1^{\text {st }}$ group that is Class II Div2 experience less pain and the severity was reduced this might me due to the forward displacement of mandible when the incisors weretorquedlabially so there was less compression on the retrodiscal tissue and hencethe pain was reduced.In group 2 Patients those subjected to Head gear experienced little pain.Thereby, the study enlightens about the relationship of orthodontics \& TMJ disorders.
\end{abstract}

\section{Introduction}

Temporomandibular joint as the name suggests is the articulation of the lower jaw(condyle of the mandible) with the cranium(inferior surface of the squamous portion of the temporal bone).It is one of the most complex joints in the body and is the articulation between the mandible and cranium. ${ }^{1}$ T.M.J. is formed by mandibular condyle fitting into the mandibular fossa of the temporal bone. Separating these two bones form direct articulation is the articular disc. Thecraniomandibular articulation is a complex synovial system composed of temporomandibular joints together with their articular ligaments and masticatory muscles. ${ }^{2}$

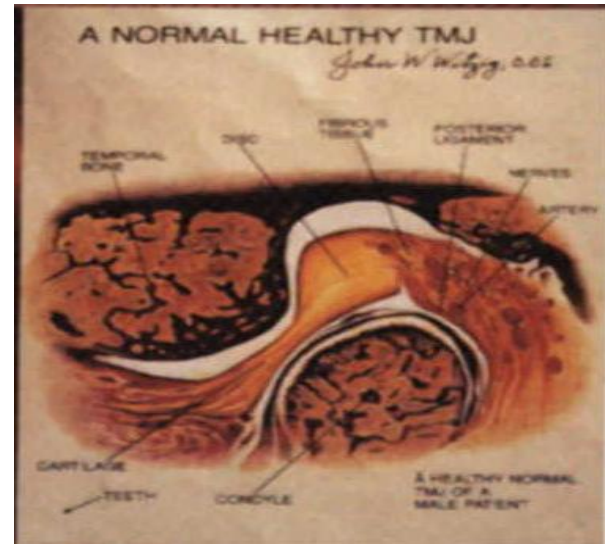

Fig.1

\section{Type of joint}

It provides for hinging movement in one place and therefore can be considered a Ginglymoid joint. At the same time, it also provides for gliding movement, whichclassifies it as Arthroidal joint. Thus it is considered asGinglymoarthroidaljointT.M.J. is classified as a compound joint. By definition a compound joint requires the presence of at least 3 bones, yet TMJ is made up of only 2 bones. Functionally, the articular disc servers as a non-ossified bone that permits the complex movements of the joint.Temporomandibular disorder, in the broad sense are to be considered acluster of joint and muscle disorders in the orofacial area, characterized primarilyby pain, joint sounds and irregular or deviating jaw function.

According to "American Academy of Orofacial Pain" - Temperomandibular disorder is a collective term embracing a number of clinical problems that involvethe masticatory musculature,temporomandibular joint and associated structures or both. ${ }^{1}$ The etiology of TMD remains mired in controversy. It is generally agreed that the etiology of symptoms of TMD is multifactorial. That is several different factors acting alone, or in varying combinations may be responsible. The orthodontist havelong been interested in the problems associated with diagnosis and management of Temperomandibular disorders. Indeed orthodontic treatment has been characterized in diverse publications as both causing and curing temperomandibular disorder. ${ }^{3}$ 
The interest of the orthodontic speciality concerning the association or lack of association between Orthodontic treatment and TMD has increased dramatically during the past decade.The attention of the orthodontic community regarding TMDhowever was heightened in the late 1980s after litigation involving the allegations that orthodontic treatment was the proximal cause of TMD in orthodontic patients. In the 1980's articles in various journals and trade magazines suggested that orthodontic treatment might play a role in initiating temperomandibular disorder.

On the other hand it was also claimed that orthodontic treatment might be effective in alleviating the signs and symptoms of TMD. The conflict became earnest when result of the famous Michigan orthodontic/TMJ law suit was announced. This litigation turned on the argument that a form of orthodontic treatment had been the cause of the patient's TMD, the six member jury were in favor of the patient. The patient named Susan Brimm, when she was 16 years began treatment to correct a Class II Div I malocclusion with a 7 $\mathrm{mm}$ of Overjet Her treatment included the removal of her upper first premolars, the use of headgear and bonding of her upper and lower teeth.

At the time the treatment was initiated she exhibited no temporomandibular symptoms. She complained that when braces were removed she suffered a severe click with severe pain and limited opening. Ultimately the matter was settled by a payment of a large sum of money to the patient. This litigious climate stimulated the American Association of Orthodontics to underwrite research concerning the relationship of orthodontic treatment to TMD. The benefits of orthodontic treatment in the management of Temperomandibular Disorder is questionable, since the occlusion is considered as having a limited role inthe cause of TMDEffect of headgear and/or class II elastics in correction of Class II malocclusions with deep interlocking cusps.

Effect of Cross elastics to correct the midline.Effect of Reverse Headgear or Class III Elastics for Correction of Class III malocclusion. And hence this study was done to confirm whether Orthodontic treatment will solve or create TMD disorders. ${ }^{10}$

\section{Objectives}

The main aim of the study is to determine, does Orthodontic treatment will cause TMD disorder or an remedy for TMD disorders.

\section{Materials And Medthods}

A Sample of 100 Patients between the age group of 10-22 yrs were taken for the Study.The samples had 60 boys and 40 girls.Following records of the patient weretaken:Lateralcephalogram,Fig (2) OPG, (Fig.3) Mounted cast (Fig.4) and Transpharyngealradiograph (Fig.5) of those patients with TMJ pain.The samples were divided into two groups depending on the symptoms andType ofmalocclusion.

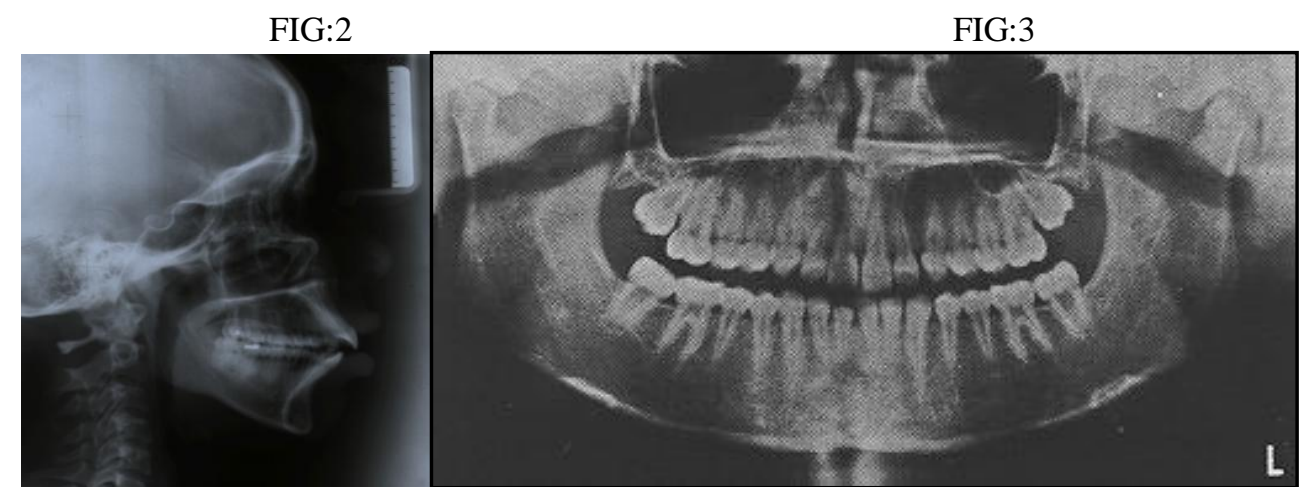

FIG:4

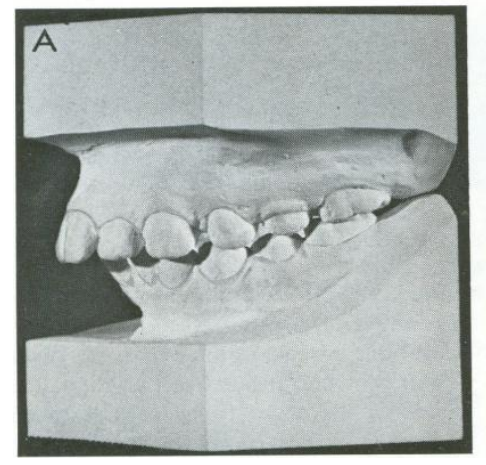

FIG:5

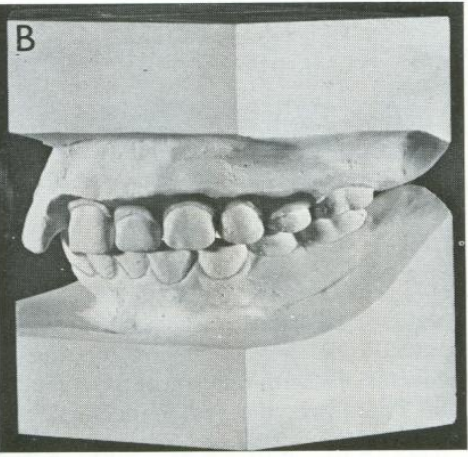

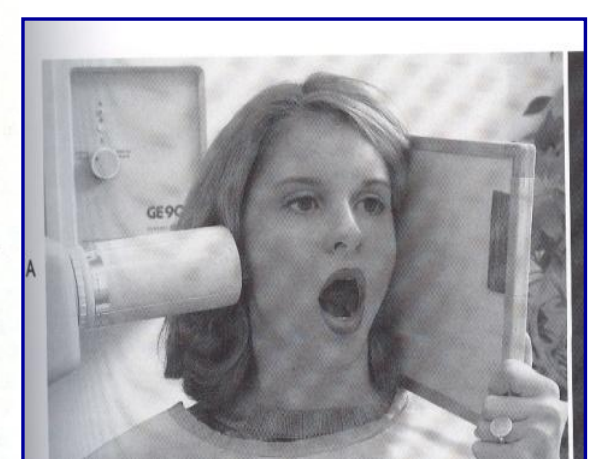


The first group consists of 50 pts with class II Div II malocclusion with mandible displaced.

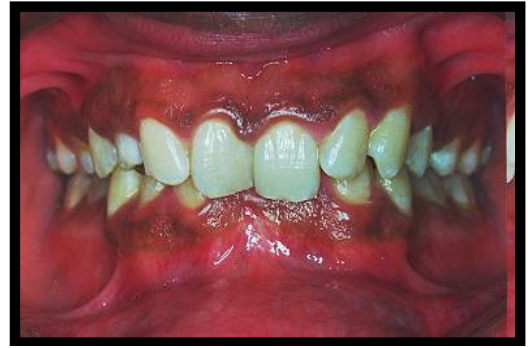

Fig .6

The second group comprised of 50 Patients of Class II Div I Malocclusion. The second had 30 patients with Maxilla Placed forward and 20 Patients with Mandible driven distally.

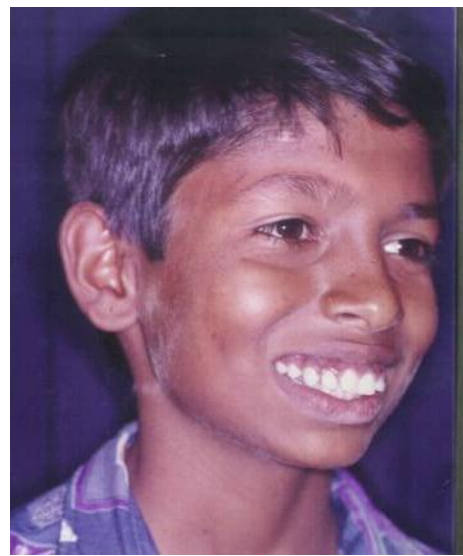

Fig.7

The treatment given varied between the two groups depending on the symtoms and type of Malocclusion. The group I patients had severe TMD disorder because of mandible driven distallythe mandible was driven distally because of the hinderence due to retroclinedIncisiors.In Group I patients and anterior Inclined Plane oraCatlans Appliance was given to remove the intrefrences and allow the mandible to move forward and to relive the symptoms of the TMJ.

In Group II patients those with Maxilla excess was given Headgear and those with Mandible deficient was given Headgear with Activator.Group II patients was free of any pain or symptom in TMJ region. The treatment was continued for $1 \mathrm{yr}$ in both the groups.

\section{Results:}

It was observed that during the course of the treatment patients of $1^{\text {st }}$ group that is Class II Div2 experience less pain and the severity was reduced this might me due to the forward displacement of mandible when the incisorsweretorquedlabially so there was less compression on the retrodiscal tissue and hencethe pain was reduced.

In group 2 Patients those subjected to Head gear experiencedlittle pain.

\section{Discussion:}

A number of viewpoint articles have asserted that a wide variety of traditional orthodontic procedures e.g. premolar extraction, extraoraltraction,retraction of maxillary anterior teeth cause TMD signs and symptoms by producing a distal displacement of condyle. Gianelly et al did the study collecting the tomograms to evaluate condylar position. They took the tomograms before orthodontic treatment in 37 consecutive patients aged 10 to 18 years and compared them with tomograms from 30 consecutively treated patients with fixed mechanotherapy and removal of four premolars.No differences in condylar position were noted between groups.

The result of thisstudy indicated that the majority of patients in Group I undergo a forward mandibular displacement and a slight opening rotation of mandible. The remainder of the samples inGroup II had distal movement of the condyle. Several view point articles including those by Roth et al and Williamson have maintained that TMDs may result from a failure to treat orthodontic patients to gnathologic standards that include the establishment of a "mutually protected occlusion" and proper seating of the mandibular condyle within the glenoid fossa. 
Research and study done by James Macnamarain 1997 revels that Orthodontic treatment are prone for TMJ disorders. But this study strongly condradicts the study as it observed that not all Orthodontictreatment are Prone for TMJ disorders but instead it very helpful in TMJ disorders has it relives the Occlusion and make the Patient free from the symptoms of TMJ.

Dibbites\& Vander weele (Netherlands) functional activator fixed appliance (Begg appliance) concluded that orthodontic treatment does not induce T.M. Dysfunction ${ }^{12}$ Hans Pancherz (AO, 2000) concluded that bite jumping with Herbst did not result in any muscular TMD, rather reduced the prevalence of capsulitis, did not induced disc displacement in subject with normal pre treatment disc position, resulted in stable repositioning of disc in subjects with partial disc displacement with reduction, but could not re capture the disc in subject with total disk displacement with or without reduction. ${ }^{14}$

Sandowsky\&BeGole did a study on the effects of Ortodontic treatment on T.M.J andconcluded from their studies that orthodontic treatment performed during adolescence did not generally increase the risk of developing T.M.D. later in life. ${ }^{\mathbf{2}}$

\section{Conclusion:}

The role of occlusion in the origin of TMJ disorders is a problem in craniofacial development. Craniofacial development is what orthodontics is all about. We orthodontists owe it to our colleagues and our patients to intensify the research in this field, for only through research will the answers be found.

\section{Bibliography}

[1]. Temperomandibular joint and masticatory muscle disorders.GeorgeA.Zarb ,BarrJ. Sessle, Gunnar E.Carlson

[2]. Fundamentals of occlusion and Temperomandibular disorders - Jeffrey P.OkesonA.Perter, SheldonG.Currntte

[3]. Temperomandibulardisorder,classification, diagnosis and management,-Weldon E.Bell.

[4]. Clinical management of Temperomandibular disorders and oro-facial .- Richard

[5]. Tempero-Mandibular disorders - Fonesca, $4^{\text {th }}$ Volume

[6]. Adult Orthodontics-DNCA

[7]. Reint M. Reynders Orthodontics and temporomandibular disorders: A review of the literature (1966-1988) AJO 1990; 97: 463-471

[8]. Sadowsky C, BeGole EA. Long-term status of temporomandibular joint function and functional occlusion after orthodontic treatment. AM J ORTHOD 1980; 78:201-12

[9]. TMJ Dysfunction and Treatment - DNCA,July 1983

[10]. Wyatt. Preventing adverse effects on TMJ through orthodontic treatment. AJO 1987; $91: 493-499$

[11]. Temperomandibular disorders and oro-facial pain. DCNA,Jan 1991

[12]. Traction, orthodontic treatment \& craniofacial dysfunction. J.M.H.Dibbets. A.J.O.D.O 1991, vol 99, 210-19

[13]. James A.McNamara,Jr., Orthodontic treatment and temperomandibulardisorders.OOO 1997;83 : 107-117

[14]. Burton H.Goldstein .Temperomandibular disorders .OOO $1999 ; 88: 379-383$

[15]. Amount \& direction of T.M.J. growth in Herbst treatment. Pancher\& Fischer.A.O.2003 vol 73;493-501 